Case I. Acute appendicitis. - May 16, 1904. Oscar M., nine years. Not feeling well for three days. Two days ago vomiting began and has since continued. Feverish and bowels sore. Examination: Tenderness over McBurney's point with moderate spasm over the entire right side and dullness everywhere. Immediate operation. Intestines collapsed and of grayish color, suggesting those of cadaver. Appendix retrocecal, slightly injected and swollen. Removed. As condition of appendix not thought to account for the alarming condition of the patient the abdomen was thoroughly explored, but nothing abnormal was found. Abdomen filled with salt solution and wicked. Patient stimulated. May 18. Vomited after operation and yesterday. Sweet odor to breath. May 22. Still occasional vomiting. May 28. Steadily failing. Pulse rising. Temperature irregularly elevated. Unable to retain anything on stomach. Died. Urine examination, May 19: No albumin or sugar; acetone and diacetic acid both present and both persisted till death.

CaSe II. Acute osteomyelitis. - April 4, 1904. Grace M. S., twelve years. Has not walked for a week because of pain in right knee. Four days ago leg and knee became swollen. Night cries, anorexia and elevated temperature for one week. Examination showed evidences of acute osteomyelitis of the lower end of the right femur which was operated upon and pus found in the medullary cavity. Following this various foci developed and the bones were trephined as follows: April 6. Right femur, pus found. April 10, left humerus, pus found. The left femur, no pus. April 13. Left fibula, pus found. April 25. Abscess of right parotid opened. After this the convalescence seemed to be going along satisfactorily, the temperature and the pulse both falling. The child then began to vomit and acetone appeared in the urine. Rectal feeding became necessary. Vomiting persisted and on May 20 , during a severe attack of vomiting, patient collapsed and died. Urine examination, May 9: Albumin and sugar absent; acetone present. From this time acetone and diacetic acid were present on every examination, May 12, 13, 14, 15, 16, 17, 18 and 19.

In the above cases some condition changed the course of the convalescence from a normal one to a critical one and finally to a fatal ending. These cases differ from others of the same disease by the presence of acetonuria, and on this, therefore, rests the blame for the deaths; whether justly or not, remains to be proved. Judging from these last cases, the finding of acetone alone or with diacetic acid in the urine seems, in the presence of symptoms of themselves alarming, to increase the gravity of the prognosis.

From a perusal of the above cases with those reported by Brewer, Brackett, Stone and Low, it seems correct to draw the following conclusions:

(1) Acetonuria is of more frequent occurrence than thought for.

(2) Its presence without symptoms has no effect on operative treatment or prognosis.

(3) Its presence with moderate symptoms is of only slight importance.

(4) Its presence with severe symptoms is of the gravest prognostic value.

Whether the severity of the symptoms on further study will be found to vary in direct proportion to the amount of acetone or diacetic acid present in the urine, I am in no position to determine.
An analysis of these cases as to age shows that acetonuria occurs more frequently in the young. By decades, the cases fall as follows: To ten years, 5 cases; ten to twenty years, 4 cases; twenty to thirty years, 3 cases; thirty to forty years, 1 case; fifty to sixty years, 2 cases. It is certainly suggestive that the two fatal cases were twelve and nine years old, and Brewer's case was twelve years old.

Brewer summarized the symptoms in his case as follows: "There suddenly occurred acute delirium, frightful hallucinations, a failure to appreciate his surroundings or recognize those about him, somnolence, coma and death within thirtytwo hours from the first untoward symptom." In the cases which I have reported, this symptom complex is met far less frequently than one characterized by vomiting. As all of the cases have occurred in hospital practice, it is possible that an initial change in the mental condition might have been overlooked. The most marked symptom, however, was the vomiting occurring without any apparent cause. The odor in the breath of acetone has been noted several times. By combining all the cases, the frequency of these prominent symptoms is found to be as follows: Vomiting: Brackett, etc., 7 cases; Boston City Hospital, 5 cases. A pathy : Brackett, etc., 1 case; Boston City Hospital, 1 case (Brewer's case). Odor of acetone in breath: Brackett, etc., 7 cases; Boston City Hospital, 2 cases (Brewer's case).

For the discussion of the nature of the condition which is accompanied by acetone and diacetic acid in the urine the reader is referred to the papers by Brewer and Brackett, Stone and Low.

The treatment mentioned several times in the reports of the cases has been the administration of bicarbonate of soda usually in doses of $30 \mathrm{gr}$. three times a day or every four hours.

\section{A CASE OF TOXIC DEGENERATION OF THE LOWER NEURONS.*}

BY JOHN E. DONLEY, M.D., PROVIDENCE.

IT is my wish in the present paper, first, to report a case of toxic degeneration of the lower spinal neurons; and, secondly, to discuss briefly some pathological and clinical resemblances between this condition on the one hand, and acute poliomyelitis, peripheral neuritis and Landry's paralysis on the other.

Miss G., age thirty, white, single, by occupation a housemaid, came under observation, complaining of weakness in the hands, forearms, legs and feet. Her family history is not significant, nor is her personal history up to an attack of what she calls " grippe," three months ago, from which she suffered for about two weeks, and from which she dates her present difficulties. About ten days after recovery from this attack she noticed that her hands were rapidly becoming weak and that she was unable to use them as formerly, on account of loss of power, more especially in those movements requiring delicate co-ordination, such, for example, as threading a needle.

About the same time she observed that her feet also *Read before the Providence Medical Society, February 1905. 
were weak, although at no time has she been incapacitated from walking. There has never been any tingling, numbness, or other paresthesia, but cramplike pains have occurred in the arms somewhat frequently.

Upon examination she was a woman of medium stature and, in general, of good development. Her pupils were equal, reacted normally to light, including the consensual reflex, and in accommodation. There were no cranial nerve anomalies. The triceps and biceps jerks were normal; patellar reactions equal and slightly exaggerated. The Achilles jerk, front tap, plantar reflex or ankle clonus could not be elicited. Objective sensory changes to touch, pain and temperature were not demonstrable, but the calf muscles were rather tender upon pressure. There had never been any sphincter trouble. The interossei, thenar and hypothenar muscles of each hand were atrophied, the wasting being about equal on the two sides; there was also some wasting and weakness of the extensors of the fingers and wrists, a tendency to drop wrist being present. In the feet the small muscles were atrophied, as were also the anterior tibial groups.

Upon electrical examination the interossei of the hands, the thenar and hypothenar muscles were unresponsive to strong faradism, and the extensors of the forearm, although contracting, required a stronger current than did the flexors. In the feet and legs the atrophied muscles contracted upon stimulation, requiring, however, a stronger current than normally. The reactions of the muscles to galvanism were not taken. Under continued treatment for seven weeks, the muscular atrophy and weakness improved very materially, and when last seen the patient looked and considered herself to be distinctly better. She was lost sight of before the ultimate result of her disease could be observed.

In a word, the important points in this case are as follows: shortly after an acute febrile attack the patient rapidly developed weakness and atrophy in the interossei, thenar and hypothenar muscles of the hands, the extensors of the wrist and fingers and the small muscles of the feet, together with the anterior tibial groups. There were no objective sensory changes and no subjective sensory symptoms, except tenderness upon pressure of the calf muscles, and cramplike pains in the arms. The condition after reaching its maximum of intensity constantly improved and when the patient was last seen this improvement was still in progress.

Writing in 1902, Dr. Stanley Barnes ${ }^{1}$ described a group of cases whose clinical features had not hitherto been recorded, and to these he gave the name of toxic degeneration of the lower neurons. Somewhat later Williamson ${ }^{2}$ published the notes of a similar case, and my own case would seem to belong in the same category. In the discussion of his cases, of which there were seven, Dr. Barnes remarks, "At the stage when they came under observation the cases recorded above all showed a well-marked atrophy of the small muscles of the hands, together with weakness of the extensors of the wrist and of the flexors of the ankle. The atrophy of the small muscles of the hand in five of the cases reached a severe degree and especially in case No. III where only the slightest contractions were possible in the interossei, lumbricales, thenar, or hypothenar muscles. In the

\footnotetext{
Stanley Barnes: Brain, vol. xxv, p. 479.
2 Williamson: Brain, vol. xxvi, p. 206.
}

more severe cases there was no reaction to the faradic current in the hand muscles, and diminished reaction in the extensor group of the forearms and in the flexor group of the ankle. In other words, there was an atrophic paralysis of the peripheral type, a paralysis in which the most distal muscles had suffered severely and in which the proximal muscles were only slightly or not at all affected. In none of the cases did the muscles particularly affected correspond to particular peripheral nerves. The distribution of atrophy corresponded rather to the segments of the cord, the first dorsal supply being especially picked out."

The truth of this last statement may be seen by reference to the case which I am reporting. It will be observed that the muscles involved are supplied by the median, musculo-spiral and ulnar nerves; but that while the distal muscles are paralyzed and atrophic, the proximal muscles supplied by these same nerves, as well as those supplied by the circumflex and musculo-cutaneous nerves, have escaped. The paralysis is of the spinal segment type, the lowest cervical and first thoracic segments, which supply the muscles of the hands, being chiefly involved.

The resemblance of these cases to peripheral neuritis is striking. There is the comparatively rapid onset, the symmetrical distribution, the tingling and numbness, the tenderness of the muscles and nerves upon pressure and the distinct muscular atrophy. The latter, however, is not in the distribution of individual peripheral nerves, but in that of spinal segments, and in this regard we observe the similarity of the disease to acute and sub-acute poliomyelitis; but here again the symmetrical distribution of the paralysis in both the upper and lower limbs and the distinct signs of peripheral nerve involvement, evidenced by the muscular tenderness, obviously exclude these cases from the class of uncomplicated poliomyelitis. Furthermore, the continuous improvement of all the affected muscles is proof in the same direction. If we reflect upon the fact that the symptom complex is consequent upon some febrile reaction, that there are signs not only of implication of the peripheral nerves, but also of the cells of the spinal cord, and that in the course of the affection there is a steady although slow improvement, I think we will be impelled to conclude that the whole neuron cell and fiber - takes part in the morbid process, and indeed of this, Dr. Barnes furnishes pathological proof in one case which he examined microscopically. We have, therefore, in toxic degeneration of the lower neurones, a symptom complex which would seem to hold a place intermediate between anterior poliomyelitis on the one side and peripheral neuritis and Landry's acute ascending paralysis on the other. The disease overlaps upon either side, and the question therefore very naturally suggests itself, Are these symptom groups - these diseases as we are accustomed to regard them - really as distinct as their separate description would lead us to believe? Are we not, perhaps, dealing with a mor- 
bid entity, essentially one, but giving rise to different symptoms according to the precise anatomical location and varying intensity of the process. A very general consensus of opinion regards the neuron as an anatomical, functional and nutritional unit; and if this is so, there would appear to be no good reason for regarding it as other than a pathological unit also. The proof, however, must come from pathology.

In the group of cases which we are considering, the cause of the degenerative change in the nervous elements is a toxin produced by bacteria in the body, or a poison introduced from without. This, circulating in the blood and vitiating the nutritive plasma which bathes the nervous tissues, must of necessity come in contact with the whole neuron; and while anatomical conditions may vary the time and degree of this contact, the general truth remains none the less true. The difficulty arises in explaining how it is, and why it is, that at one time the axon or segments of it, and at another time the cell body, and at still another the whole neuron appears to be chiefly affected.

From the pathological side we may first consider alcoholic multiple neuritis. There are few who maintain at the present time that alcoholic neuritis is an inflammatory condition of the nerves. It is essentially a degeneration of nerve fibers, as is plainly evident from the study of acute cases such as those of Sydney Cole, ${ }^{3}$ Howard Tooth and Sidney Martin. Proliferation of neurilemma nuclei and connective tissue cells, emigration of leucocytes, and formation of new fibrous tissue do indeed occur, but these are certainly secondary in point of time, and the primary process is one of fiber degeneration. Not only are the fibers as such affected, but the anterior horn cells undergo a typical Nissl degeneration which has been described and pictured by Achard and Soupault, Ballet and Dutil, Dejerine and Thomas, Marinesco, Larkin and Jelliffe, Mott, Wright and Orange, and others.

The pathological condition, therefore, in alcoholic and other forms of toxic peripheral neuritis is not a neuritis at all, and is not strictly limited to the peripheral nerve fiber. It is a degeneration affecting the whole neuron - both cell and fiber - although different degrees of involvement of each must be conceded.

The same remarks apply with equal force to acute anterior poliomyelitis of infants and adults. In a case of this disease recently reported by Dr. F. W. Mott, ${ }^{4}$ the spinal cord, peripheral nerves and muscles were examined after the fatal termination sixteen days from the onset. The patient was a child, aged five months. Postmortem the degenerative process had implicated both the periphereal nerves and the cells of the spinal cord, and furthermore had produced acute fatty changes in the muscles.

More interesting still than poliomyelitis, peripheral neuritis or toxic degeneration of the lower neurons is Landry's ascending paralysis; for

3 Cole: Brain, vol. xxv, p. 326.

Mott: Archives of Neurology, London County Asylum, vol. i. in the microscopic findings in this disease we obtain important evidence as to the pathological unity of the neuron. Excluding the very rare and constantly diminishing cases in which no pathological changes are to be found, and considering only those in which definite post-mortem evidence of the disease exists, we find according to Farquhar Buzzard, ${ }^{5}$ that a certain number have been remarkable only for the signs of degeneration in the peripheral nerves, and on account of the absence or scarcity of cell changes in the cord, have been held to prove the peripheral origin of the disease. A majority of recent cases, however, have shown significant changes in the nerve cells of the cord, and especially in those of the anterior horns, and this condition has often been associated with early myelin degeneration, both in the peripheral nerves and in the white matter of the central nervous system. The intensity of the process as it affects the cellular elements has been of all degrees, and has varied from a condition which could hardly be regarded as abnormal to one which has closely resembled an acute anterior poliomyelitis. Because of these pathological findings, Buzzard regards the intoxication as affecting all parts of the central and peripheral nervous system, but with an intensity which varies with the susceptibility of the different neurons to the poison, and with the energy and duration of its activity.

Additional confirmation of my contention that these different clinical types are in reality but varied aspects of one fundamental disease process, is derived from those cases, not uncommon in the literature, where two or more diseases are present together or follow upon one another in which latter case we call them complications of the primary difficulty. Commenting upon the pathological investigation of one of his fatal cases of toxic degeneration of the lower neurons, Stanley Barnes observes, "There can be little doubt that this patient succumbed to the third attack of a toxemia which caused degeneration of the lower neurons. I would again emphasize the remarkable similarity between the terminal attack and the condition seen in Landry's paralysis. If this patient had come into the hospital for the first time during the final acute attack, and had shown no signs of previous nervous disease, there can be no doubt that a diagnosis of Landry's paralysis would have been made without hesitation."

After describing a case of puerperal polyneuritis in which the primary symptoms were followed by signs of spinal cord involvement, and in which both peripheral nerves and spinal cord cells were found degenerated post-mortem, Dr. James Stewart ${ }^{6}$ writes as follows: "The clinical course makes it highly probable that we had to deal, first with a neuritis, and later with a localized myelitis (poliomyelitis). The symptoms were for several months those of a neuritis rather than a poliomyelitis. In fact, at no time were there sufficiently distinctive symptoms present to enable

S Buzzard: Brain, vol. xxvi, p. 94.

Stewart: Phila. Med. Jour., May, 1801. 
one to say definitely that the spinal cord was involved. It was only the gradual ascending character of the paralysis (Landry type) that some three or four months before death gave a clew as to a probable spinal involvement. The development of the symptoms and the appearances met with in the nerves make it clear that we had to do in the first place with a parenchymatous neuritis. The prolonged primary stage of numbness in all the four extremities, together with a prolonged period of simple weakness of the peripheral muscles, pointing to a distal parenchymatous multiple neuritis as the primary lesion."

It is cases such as these, cases which do not fit under the description of any of the well-defined clinical groups, which suggest the underlying common relation of all.

An analogy may be drawn from hysteria and neurasthenia. While it is comparatively easy to separate precisely those cases at the extreme ends of the series, nevertheless as we approach the center, such a distinction becomes oftentimes practically impossible, and we are driven to the necessity of speaking of hystero-neurasthenia. For similar reasons, after reviewing the clinical and pathological findings in the cases under description, I think the following conclusions have at least a tentative justification:

First: Toxic degeneration of the lower neurons, acute anterior poliomyelitis, peripheral neuritis and Landry's paralysis are essentially degenerative conditions of nervous elements.

Second: The exciting cause of this degeneration is a toxemia, which may be the result of bacteria, auto-toxins, or poisons introduced from without; and,

Third: The whole neuron, both cell and fiber, suffers in every case, the clinical symptoms, however, depending upon the intensity, the duration, and the anatomical situation of the morbid process.

\section{Elinical gDepartment.}

\section{FROM THE WELD WARD FOR DISEASES OF THE SKIN. MASSACHUSETTS GENERAL HOSPITAL. \\ Service of Charles J. White, M.D. BY FREDERICK \&. BURNS, M.D., Assistant Physician for Diseases of the Skin.}

DERMATITIS VENENATA FROM MOSQUITOES.

MARY H., age twenty-four. Born in Ireland, a recent immigrant.

Three weeks after arrival in this country the patient went into domestic service in a neighboring suburban town, where mosquitoes abounded. After being bitten on the exposed portions of the skin, these parts swelled and became so inflamed that relief was sought at the hospital.

The patient was a healthy, well-developed Irish girl. On the parts generally that had been bitten the skin was highly erythematous, swollen and covered with numerous large wheals, many of which were distinctly vesicular and bullous. Swelling of the eyelids was sufficiently intense to close the eyes. There was slight fever and malaise.
Under local applications of oxide of zinc and lime water, and free saline catharsis, the process subsided, and in a week's time the patient was discharged well.

Arrived recently from a portion of Europe where mosquitoes are not indigenous, and therefore unaccustomed to their bites, the patient experienced the effect of an entirely new poison in her skin, which, combined with the contributing influences of a steerage passage across the Atlantic, explained the unusual severity of the cutaneous reaction.

[J. C. White (Journ. Cutaneous Dis., Oct., 1890, Dermat. venenata, 1887) some years ago called attention to this variety of dermatosis, and cited numerous instances in his experience of the extraordinary effect of mosquitoes and certain vegetable poisons on the skins of immigrants who, before arriving in this country, had never been exposed to these irritants.]

\section{ERYTHEMA MULTIFORME PSORIASIS.}

Margaret W., age twenty, laundress. Born in Ireland. Family history unimportant. Previous health of patient always good.

The present illness began three days ago, when the ankles swelled and became painful in the evening. The following morning both legs were swollen, reddened and painful. The lesions on the face were noticed two days ago.

On entrance to the ward both legs were edematous and pitted on pressure. Over the surface of the legs generally, and most numerous anteriorly, were red, cent to half dollar sized, deeply seated nodules, decidedly painful on pressure. The backs of the hands presented a few, bright red, large bean-sized papules with several peripherally-situated vesicles. The bridge of the nose also showed two papulo-vesicular lesions.

During the first five days in the hospital there were evening exacerbations of temperature, subsiding to normal on the sixth day. The general physical examination of the patient was quite negative.

In addition to the acute erythematous affection, there was a generalized, sparsely disseminated papulosquamous psoriasis of six months' duration.

\section{RAYNAUD'S DISEASE.}

Tilly H., age forty-four. Born in Austria. General family and personal history unimportant.

The present illness began four years ago, when the fingers of both hands began gradually to get blue and cold, especially in winter, and to be accompanied by a burning pain. The feet have never been affected. The ears seem sensitive at times.

Both hands felt decidedly cool, getting colder towards the ends of the fingers. The skin of the fingers was waxy, pinkish-white in hue, with a marked atrophic appearance. The finger-nails were either entirely atrophied or partially so, with thickening and transverse striation. At the tip of the second finger of the left hand was an open, superficial, sluggish ulceration. The radial pulse was regular, small and of poor tension. (78 $\mathrm{m} \mathrm{Hg}$.) Nerve reactions were normal. Under tonic treatment the general condition of the patient improved considerably and the local affection somewhat.

\section{PEMPHIGUS FOLIACEUS.}

S. M., infant, female, ten days old. Born in Dorchester.

Family history. - Infant's mother has two older children, both of whom have always been well. No history of miscarriages in the mother, nor of venereal nor cutaneous disease in either parent. 